\title{
Profile of Anisometropia and Aniso-Astigmatism in Children: Prevalence and Association with Age, Ocular Biometric Measures, and Refractive Status
}

\author{
Lisa O'Donoghue, ${ }^{1}$ Julie F. McClelland, ${ }^{1}$ Nicola S. Logan, ${ }^{2}$ Alicja R. Rudnicka, ${ }^{3}$ Chris G. Owen, ${ }^{3}$ \\ and Katbryn J. Saunders ${ }^{1}$
}

Purpose. We describe the profile and associations of anisometropia and aniso-astigmatism in a population-based sample of children.

Methods. The Northern Ireland Childhood Errors of Refraction (NICER) study used a stratified random cluster design to recruit a representative sample of children from schools in Northern Ireland. Examinations included cycloplegic (1\% cyclopentolate) autorefraction, and measures of axial length, anterior chamber depth, and corneal curvature. $\chi^{2}$ tests were used to assess variations in the prevalence of anisometropia and anisoastigmatism by age group, with logistic regression used to compare odds of anisometropia and aniso-astigmatism with refractive status (myopia, emmetropia, hyperopia). The MannWhitney $U$ test was used to examine interocular differences in ocular biometry.

Results. Data from 661 white children aged 12 to 13 years (50.5\% male) and 389 white children aged 6 to 7 years (49.6\% male) are presented. The prevalence of anisometropia $\geq 1$ diopters sphere (DS) did not differ statistically significantly between 6- to 7-year-old (8.5\%; $95 \%$ confidence interval $[\mathrm{CI}], 3.9-13.1)$ and 12 - to 13 -year-old $(9.4 \%$; $95 \% \mathrm{CI}$, 5.9-12.9) children. The prevalence of aniso-astigmatism $\geq 1$ diopters cylinder (DC) did not vary statistically significantly between 6- to 7 -year-old $(7.7 \%$; $95 \% \mathrm{CI}, 4.3-11.2)$ and 12 - to 13-year-old (5.6\%; 95\% CI, 0.5-8.1) children. Anisometropia and aniso-astigmatism were more common in 12- to 13-yearold children with hyperopia $\geq+2$ DS. Anisometropic eyes had greater axial length asymmetry than nonanisometropic eyes. Aniso-astigmatic eyes were more asymmetric in axial length and corneal astigmatism than eyes without anisoastigmatism.

Conclusions. In this population, there is a high prevalence of axial anisometropia and corneal/axial aniso-astigmatism,

From the ${ }^{1}$ Vision Science Research Group, School of Biomedical Sciences, University of Ulster, Coleraine, Northern Ireland, United Kingdom; the ${ }^{2}$ Ophthalmic Research Group, Aston University, Birmingham, United Kingdom; and the ${ }^{3}$ Division of Population Health Sciences and Education, St. George's, University of London, London, United Kingdom.

Supported by a research scholarship from the College of Optometrists, London, United Kingdom (the NICER study).

Submitted for publication October 2, 2012; revised November 29, 2012; accepted December 5, 2012.

Disclosure: L. O'Donoghue, None; J.F. McClelland, None; N.S. Logan, None; A.R. Rudnicka, None; C.G. Owen, None; K.J. Saunders, None

Corresponding author: Lisa O'Donoghue, Vision Science Research Group, School of Biomedical Sciences, University of Ulster, Cromore Road, Coleraine, Northern Ireland, BT52 1SA, UK; 1.odonoghue@ulster.ac.uk. associated with hyperopia, but whether these relations are causal is unclear. Further work is required to clarify the developmental mechanism behind these associations. (Invest Ophthalmol Vis Sci. 2013;54:602-608) DOI:10.1167/ iovs. 12-11066

nisometropia is an interocular difference in refraction that $\mathbf{A}_{\text {can }}$ be associated with significant visual problems, including aniseikonia, strabismus, and reduced stereopsis. ${ }^{1-3}$ It also is recognized widely as an amblyogenic risk factor, ${ }^{4}$ with a greater magnitude of anisometropia being associated with more severe amblyopia. ${ }^{3,5}$

The prevalence of anisometropia decreases typically during the first year of life. ${ }^{6,7}$ Beyond this early period, several studies have reported that prevalence is relatively stable in early ${ }^{8-10}$ and late childhood. ${ }^{10,11}$ Other studies have reported an increase in prevalence throughout childhood associated with myopia $^{12,13}$ and hyperopia. ${ }^{12}$ Describing the prevalence of anisometropia in childhood is complicated further as it may be population-specific, with reports of a prevalence $(\geq 1.00$ diopters sphere [DS]) of $1.6 \%$ in 6-year-old children in Australia, ${ }^{14} 6.7 \%$ in 4 - to 13 -year-old American Indians,,${ }^{11}$ and $9.9 \%$ in 7 - to 18 -year-olds in Taiwan. ${ }^{15}$ Whether these differences are genetic or geographic in nature, or merely reflect the different ages of the children or the differing prevalence of myopia and hyperopia in these populations is unclear

Few studies have examined interocular differences in astigmatism (aniso-astigmatism), but of those that have, the prevalence of aniso-astigmatism $(\geq 1.00$ diopters cylinder [DC]) also varies widely from $1 \%$ in 6 -year-old children in Australia ${ }^{14}$ and 3.3\% in 6- to 11-year-olds in Japan ${ }^{16}$ to $15 \%$ in 4- to 13 -yearold American Indian children where there also is a high prevalence of astigmatism and hyperopia. ${ }^{11}$

Little is known about either the prevalence of anisometropia or aniso-astigmatism in childhood in Northern Ireland, where there is a high prevalence of refractive errors (myopia, hyperopia, and astigmatism) compared to similarly-aged white children elsewhere. ${ }^{17-20}$ Understanding the prevalence of anisometropia and aniso-astigmatism, and their associations with refraction and age may be useful in designing programs to prevent amblyopia, and assist in deciding when and how to implement such programs. ${ }^{15}$

This study describes the prevalence of anisometropia and aniso-astigmatism in 6- to 7-year-old and 12- to 13-year-old white children in Northern Ireland, UK, and explores the associations with refractive error and ocular biometric parameters. The relation between binocular vision status and anisometropia is also explored. 


\section{METHODS}

The Northern Ireland Childhood Errors of Refraction (NICER) study is a population-based survey of the refractive status of school children living in Northern Ireland. The study was approved by the University of Ulster Research Ethics Committee and the conduct of the study adhered to the tenets of the Declaration of Helsinki. After an explanation of the nature and possible consequences of the study, written consent was obtained from a parent/guardian of all children before the examination. The 12- to 13-year-old children also gave written consent, while verbal assent was obtained from the 6-to 7-yearold children.

The study methods have been described in detail previously. ${ }^{21}$ In brief, stratified random sampling of schools from geographic areas characteristic of Northern Ireland was used to obtain a representation of schools and children from urban/rural and deprived/nondeprived areas. Within individual schools, all children in one or more classes were invited to participate. Potential participants were aged 6 to 7 years and 12 to 13 years. The protocol for data collection included measurement of logMAR monocular distance visual acuity from both eyes, uncorrected and presenting (with spectacles if worn); assessment of ocular posture at distance and near using a cover/uncover test; and cycloplegic autorefraction ( $1 \%$ cyclopentolate hydrochloride following $0.5 \%$ proxymetacaine, both Minims single dose; Bausch \& Lomb, Rochester, NY) using a binocular open-field autorefractor (Shin-Nippon SRW-5000; Shin-Nippon Machinery Company, Ltd., Tokyo, Japan). At least five measurements were taken, with the representative value as determined by the instrument used in subsequent analyses. Following cycloplegia, the Zeiss IOLMaster (Carl Zeiss, Jena, Germany) was used to take ocular biometric measures: at least three measurements of axial length, five simultaneous anterior chamber depth (ACD) measurements, and three corneal radius of curvature readings. Participants were tested within school premises during the school day, between May 2006 and March 2008.

\section{Definitions}

Anisometropia is defined as the absolute interocular difference in spherical equivalent refractive error (SER, sphere $+1 / 2$ cylinder). Anisoastigmatism is defined as the absolute interocular difference in refractive astigmatism. To facilitate comparisons with other populations, prevalence of anisometropia and aniso-astigmatism of $\geq 1.00$ diopters (D) are presented. ${ }^{14}$

To enable the description of the prevalence of anisometropia and aniso-astigmatism in differing refractive conditions, subjects were classified using SER as myopic if they have -0.50 DS or more myopia in either eye. Any remaining subjects then were categorized as having moderate hyperopia if there was at least $+2.00 \mathrm{DS}$ in either eye, or low hyperopia (at least +0.50 DS and $<+2.00$ DS in either eye) with the final classification being emmetropia $(>-0.50$ DS and $<+0.50$ DS in both eyes). ${ }^{22}$ Therefore, it was not possible for emmetropic subjects to be anisometropic.

Binocular vision status was recorded using the results of the cover/ uncover test at distance, as either orthophoric, heterophoric (including exophoria, esophoria, and hyperphoria), esotropic, or exotropic. Where hypertropia was present $(n=1)$ classification was made on the basis of the concurrent horizontal tropia.

\section{Statistics}

All statistical analyses were done using Intercooled Stata 11 (StataCorp, College Station, TX). The Mann-Whitney $U$ test was used to explore differences in the distribution of anisometropia and anisoastigmatism by age group. Prevalence results were adjusted for the cluster design. The $\chi^{2}$ test was used to assess variations in the prevalence of anisometropia ( $\geq 1.00$ DS) and aniso-astigmatism ( $\geq 1.00$ DC) by age group. Logistic regression was used to compare the odds of anisometropia $\geq 1 \mathrm{D}$ in low and moderate hyperopia compared to myopia, and the odds of aniso-astigmatism $\geq 1 \mathrm{D}$ in low and moderate hyperopia, and myopia compared to emmetropia, taking account of the clustered design. The Mann-Whitney $U$ test was used to examine interocular difference in ocular biometry and acuity in children with and without anisometropia, and with or without aniso-astigmatism. Spearman's rank correlation was used to assess the strength of the association between the interocular difference in ocular biometric measures and the amount of anisometropia and aniso-astigmatism. Fisher's exact test was used to assess the difference in binocular vision status with age group. All confidence intervals (CIs) are 95\%. Results were considered statistically significant if $P<0.05$.

\section{Results}

Of the children invited to participate in the study, parental consent was obtained from $62 \%$ of 6 - to 7 -year-olds and $65 \%$ of 12- to 13-year-olds. Reflective of the Northern Irish population, $98.7 \%$ of participants were white, and this report presented data from 389 white children aged 6 to 7 years (49.6\% male) and 661 white children aged 12 to 13 years $(50.5 \%$ male) for whom data collection was complete from both eyes (99.7\%). The mean ages of the two study groups were 7.1 years (range $6.3-7.8$ years) and 13.1 years (range 12.1-14.1 years), respectively.

\section{Distribution of Anisometropia and Aniso- Astigmatism}

Most of the anisometropia and aniso-astigmatism was of a low degree. There were no statistically significant age group differences in the distribution of anisometropia or anisoastigmatism (both $P>0.42$ ): the median level of anisometropia in both age groups was $0.25 \mathrm{D}$ (interquartile range [IQR] $0.125-0.50$ ), while the median level of aniso-astigmatism in both age groups was $0.25 \mathrm{D}$ (IQR $0.00-0.50$ ).

\section{Prevalence of Anisometropia and Aniso- Astigmatism}

In the younger age group the prevalence of myopia was $2.8 \%$ (95\% CI, 1.3-4.3) and the prevalence of moderate hyperopia $\geq+2$ DS was $26.5 \%$ (95\% CI, 19.8-33.2). In 12- to 13 -year-old children the prevalence of myopia was $17.7 \%$ (95\% CI, 13.2$22.2)$ and that of moderate hyperopia $\geq+2$ DS was $14.7 \%(95 \%$ CI, 9.9-19.4).

The prevalence of anisometropia $\geq 1$ DS did not differ statistically significantly between 6- to 7-year-old (8.5\%; $95 \% \mathrm{CI}$, 3.9-13.1) and 12- to 13 -year-old (9.4\%; 95\% CI, 5.9-12.9; $P=$ 0.62 ) children. The prevalence of aniso-astigmatism $\geq 1$ DC also did not vary statistically significantly between 6 - to 7 -yearold $(7.7 \%$; $95 \%$ CI, 4.3-11.2) and 12- to 13 -year-old $(5.6 \% ; 95 \%$ CI, 3.0-8.2; $P=0.18$ ) children.

Figure 1 shows the prevalence of anisometropia by refractive status.

In the younger age group, there was no evidence of any difference in the prevalence of anisometropia by refractive classification. The 95\% CIs in these instances were wide and encompassed an odds ratio (OR) of 1.0 (low hyperopia versus myopia: OR 0.3 ; 95\% CI, 0.04-3.4; moderate hyperopia versus myopia: OR 3.0; 95\% CI, 0.4-22.0). In the older age group, the odds of anisometropia was $80 \%$ lower in children with low level of hyperopia compared to myopia (OR 0.2 ; 95\% CI, 0.1 $0.5 ; P<0.001)$. However, moderate hyperopia was associated with approximately 8 times the odds of anisometropia compared to myopia (OR 8.0; 95\% CI, 4.4-14.6; $P<0.001$ ).

Figure 2 shows the prevalence of aniso-astigmatism by refractive status. 

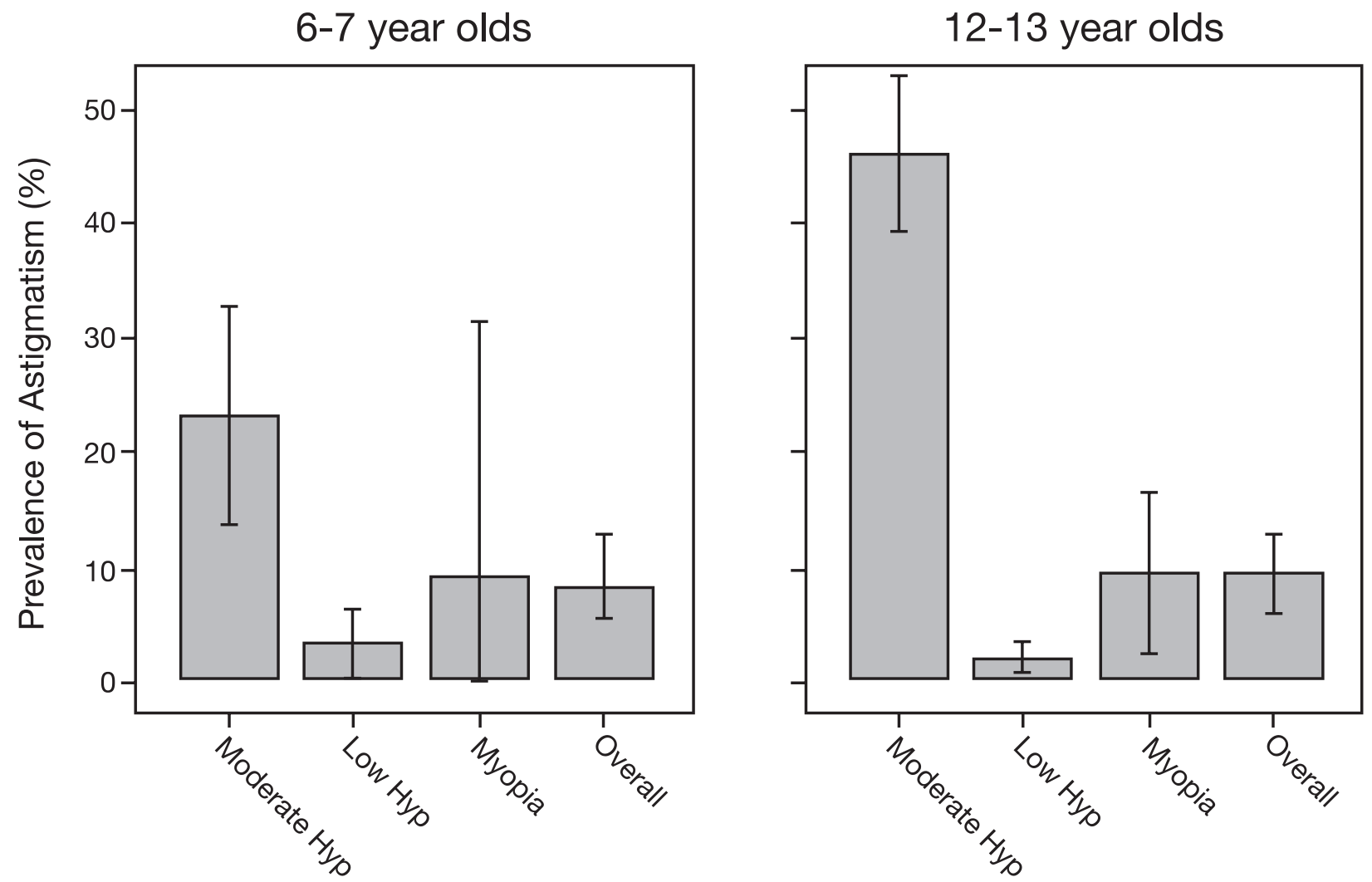

FigURE 1. Prevalence of anisometropia ( $>1$ DS) with refractive classification. Myopia $<-0.50$ DS, low hyperopia (Low Hyp) $>+0.50$ DS and $<+2.00$ DS, moderate hyperopia (Moderate Hyp) $\geq+2.00$ DS. Emmetropic children could not be defined as anisometropic. The error bars illustrate the $95 \%$ CI.
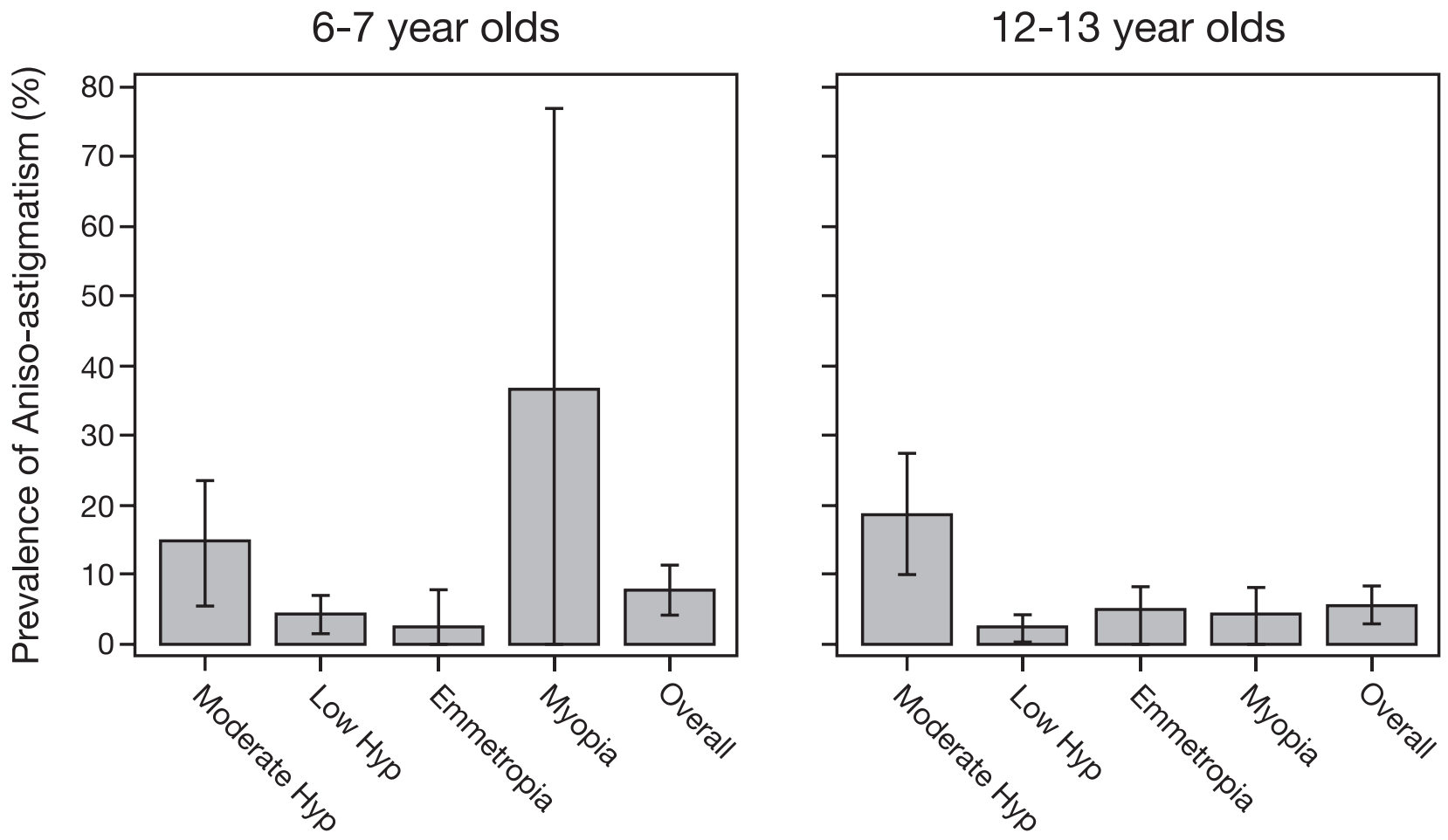

Figure 2. Prevalence of aniso-astigmatism ( $\geq 1$ DC) with refractive classification. Myopia $\leq-0.50$ DS, emmetropia $>-0.50$ DS and $<+0.50$ DS, low hyperopia (Low Hyp) $\geq+0.50$ DS and $<+2.00$ DS, moderate hyperopia (Moderate Hyp) $\geq+2.00$ DS. The error bars illustrate the $95 \%$ CI. 
TABLE 1. The Mean Interocular Difference in Ocular Biometric Parameters in Subjects with and without Anisometropia

\begin{tabular}{|c|c|c|c|c|}
\hline & \multicolumn{4}{|c|}{ Mean Interocular Difference, $\mathrm{mm}( \pm \mathrm{SD})$} \\
\hline & \multicolumn{2}{|c|}{ Age 6 to 7 Years } & \multicolumn{2}{|c|}{ Age 12 to 13 Years } \\
\hline & $\begin{array}{c}\text { Anisometropia } \geq 1 \mathrm{D} \\
(n=33)\end{array}$ & $\begin{array}{c}\text { Anisometropia }<1 \text { D } \\
(n=356)\end{array}$ & $\begin{array}{c}\text { Anisometropia } \geq 1 \mathrm{D} \\
(n=62)\end{array}$ & $\begin{array}{c}\text { Anisometropia }<1 \mathrm{D} \\
(n=559)\end{array}$ \\
\hline Axial length & $0.40( \pm 0.39)$ & $0.10( \pm 0.10)$ & $0.60( \pm 0.50)$ & $0.10( \pm 0.08)$ \\
\hline $\mathrm{ACD}$ & $0.10( \pm 0.23)$ & $0.10( \pm 0.23)$ & $0.10( \pm 0.24)$ & $0.13( \pm 0.29)$ \\
\hline Corneal curvature & $0.04( \pm 0.04)$ & $0.05( \pm 0.05)$ & $0.05( \pm 0.04)$ & $0.04( \pm 0.04)$ \\
\hline
\end{tabular}

There was no clear evidence of a difference in the odds of aniso-astigmatism between emmetropes and hypermetropes in the younger age group. Although the risk of aniso-astigmatism appeared to be higher in myopes than in emmetropes, these results must be interpreted with caution due to the small number of cases by refractive classification giving rise to very wide CIs for the younger age group (OR 21.1; 95\% CI, 1.8-249; $P=0.015$ ). Also, of the 11 myopic 6- to 7-year-olds, all four who had aniso-astigmatism were classified as myopic due to the effect of unilateral astigmatism on the SER. In the older age group, children with a moderate level of hyperopia were four times more likely than emmetropes to exhibit aniso-astigmatism (OR 4.1; 95\% CI, 1.6-10.1;P=0.003). There was no evidence of a difference in the occurrence of aniso-astigmatism between the other two refractive groups and emmetropia (both $P>0.14$ ).

\section{Relation between Anisometropia and Aniso- Astigmatism, and Ocular Biometric Parameters}

There was a statistically significant variation in interocular difference in axial length (but not ACD or mean corneal curvature) in subjects with anisometropia $\geq 1$ DS compared to those with $<1$ DS anisometropia in both age groups (both $P$ $<0.001$, Table 1).

There was an association between the degree of anisometropia and the interocular difference in axial length (Spearman's correlation $=0.19$ for 6 - to 7 -year-olds and 0.26 for 12 - to 13-year-olds, both $P<0.001)$ but not with interocular difference in ACD or corneal curvature (all $P>0.17$ ).

In both age groups there was a statistically significant variation in interocular difference in axial length and corneal astigmatism (but not ACD) in subjects with aniso-astigmatism $\geq 1$ DC compared to those with aniso-astigmatism $<1$ DC (all $P$ $<0.02$, Table 2).

There was a statistically significant association between the degree of aniso-astigmatism and interocular difference in corneal astigmatism in 6- to 7-year-olds (Spearman's correlation $=0.11, P=0.03$ ) but not 12 - to 13 -year-olds (Spearman's correlation $=0.04, P=0.33$ ). Although there was no statistically significant association between degree of anisoastigmatism and interocular difference in axial length in 6- to 7- year-olds (Spearman's correlation $=0.05, P=0.36$ ), there was in 12- to 13-year-olds (Spearman's correlation $=0.10, P=0.01$ ). There was no statistically significant association between degree of aniso-astigmatism and interocular difference in ACD in either age group (both $P>0.90$ ).

\section{Relation between Anisometropia and Aniso- Astigmatism, and Visual Acuity}

There was a statistically significant difference between subjects with and without anisometropia and aniso-astigmatism in terms of their uncorrected and presenting (with spectacles if worn and available) interocular logMAR acuity differences (Table 3, all $P<0.04$ ).

\section{Relation between Anisometropia and Binocular Vision Status}

There was no statistically significant difference in binocular vision status between the two age groups $(P=0.92$, Table 4$)$.

Although the number of children with a binocular vision anomaly was small, binocular vision defects were associated with anisometropia $\geq 1$ DS: of the 6- to 7-year-olds, two (25\%) of those with esotropia, two (50\%) with exotropia, two (12.5\%) with a phoria, and $27(7.4 \%)$ who were orthophoric had anisometropia; of the 12- to 13 -year-olds, $44 \%$ of those with esotropia $(n=7)$ had anisometropia compared to $40 \%(n=2)$ of those with exotropia, $17 \%(n=5)$ with a phoria and $7.9 \%(n$ $=48$ ) who were orthophoric.

\section{Discussion}

Our study describes, for the first time to our knowledge, the prevalence of anisometropia and aniso-astigmatism in Northern Irish school children. While a prevalence of anisometropia $(9.3 \%)$ similar to that found in our study has been reported by Shih et al. in a group of Taiwanese school children, ${ }^{15}$ the high prevalence of anisometropia in Taiwan was attributed to the associated high prevalence of myopia in the study population, whereas in our study anisometropia at age 12 to 13 years was more common in children with moderate hyperopia than in

TABLE 2. The Mean Interocular Difference in Ocular Biometric Parameters in Subjects with and without Aniso-Astigmatism

Mean Interocular Difference $( \pm$ SD)

Age 6 to 7 Years

\begin{tabular}{lcc}
\cline { 2 - 3 } & \multicolumn{2}{c}{ Age 6 to 7 Years } \\
\cline { 2 - 3 } & $\begin{array}{l}\text { Aniso-Astigmatism } \geq \mathbf{1} \mathrm{D} \\
(\boldsymbol{n}=30)\end{array}$ & $\begin{array}{c}\text { Aniso-Astigmatism }<\mathbf{1} \text { D } \\
(\boldsymbol{n}=359)\end{array}$ \\
\hline Axial length, mm & $0.28( \pm 0.38)$ & $0.11( \pm 0.13)$ \\
ACD, mm & $0.10( \pm 0.24)$ & $0.10( \pm 0.23)$ \\
Corneal astigmatism, D & $0.83( \pm 0.76)$ & $0.28( \pm 0.28)$ \\
\hline
\end{tabular}

Axial length, mm

ACD, mm

Corneal astigmatism, D
Age 12 to 13 Years

Aniso-Astigmatism $\geq 1$ D Aniso-Astigmatism $<1$ D $(n=62) \quad(n=559)$

$\begin{array}{ll}0.33( \pm 0.47) & 0.14( \pm 0.20) \\ 0.11( \pm 0.24) & 0.13( \pm 0.29) \\ 0.76( \pm 0.77) & 0.33( \pm 0.31)\end{array}$


TABLE 3. Interocular LogMAR Acuity Differences (Uncorrected and Presenting) between Subjects with and without Anisometropia and AnisoAstigmatism

\begin{tabular}{|c|c|c|c|c|}
\hline & \multicolumn{4}{|c|}{ Average Interocular Acuity Differences LogMAR ( \pm SD) } \\
\hline & \multicolumn{2}{|c|}{ Uncorrected } & \multicolumn{2}{|c|}{ Presenting } \\
\hline & 6 to 7 Years & 12 to 13 Years & 6 to 7 Years & 12 to 13 Years \\
\hline Anisometropia $\geq 1 \mathrm{D}$ & $0.20( \pm 0.25)$ & $0.20( \pm 0.24)$ & $0.15( \pm 0.20)$ & $0.14( \pm 0.20)$ \\
\hline Anisometropia $<1 \mathrm{D}$ & $0.04( \pm 0.07)$ & $0.05( \pm 0.09)$ & $0.03( \pm 0.06)$ & $0.05( \pm 0.08)$ \\
\hline Aniso-astigmatism $\geq 1 \mathrm{D}$ & $0.17( \pm 0.25)$ & $0.16( \pm 0.23)$ & $0.11( \pm 0.18)$ & $0.13( \pm 0.21)$ \\
\hline Aniso-astigmatism $<1 \mathrm{D}$ & $0.04( \pm 0.08)$ & $0.06( \pm 0.11)$ & $0.04( \pm 0.07)$ & $0.05( \pm 0.09)$ \\
\hline
\end{tabular}

those with myopia. A lower prevalence of anisometropia of $5.8 \%$ was reported in children in the United States aged 12 to 15 years where anisometropia was associated with myopic and hyperopic refractive errors. ${ }^{12}$ However, the US study was not population-based and relied on non-cycloplegic measures of refractive error, so comparisons with our study are not straightforward. A further study of a UK adult population supports an association with ametropia (myopia and hyperopia). ${ }^{23}$ It may be that our study participants were too young to demonstrate an association with myopia, which may become evident as the study population ages and more myopia emerges.

In our study aniso-astigmatism appeared to be associated with myopia in the younger age group and with moderate hyperopia in the older age group. However, the association with myopia must be treated cautiously as this was not found in the older age group and the prevalence of myopia was low in 6- to 7-year-olds. SER also has been used to classify refractive status and all four myopic 6- to 7-year-old subjects with anisoastigmatism had been classified as myopic due to astigmatism in one eye.

White school children in Australia, where the prevalence of hyperopia and astigmatism is much lower, ${ }^{17,22,24}$ have a much lower prevalence of anisometropia (1.6\%) and aniso-astigmatism $(0.8 \%)$ than similarly-aged white school children in our study. Support for an association between aniso-astigmatism and hyperopia comes from a study of American Indian children who have a high prevalence of aniso-astigmatism (15\%) associated with hyperopia and astigmatism. ${ }^{11}$

There was no significant difference in the distribution or prevalence of anisometropia or aniso-astigmatism between the two age groups. Previous reports on the prevalence of anisometropia with age have been equivocal, with some studies reporting no association ${ }^{14,16}$ and others reporting an increased prevalence of anisometropia with age. ${ }^{12,15,25,26}$ Many studies that show increasing prevalence with age have been done on populations with a high prevalence of myopia, the prevalence of which also is known to increase with age. Although the cross-sectional data from our study have shown that the prevalence of anisometropia did not vary statistically significantly between these two age groups, we cannot conclude that anisometropia remains stable throughout childhood or at an individual level. Much of the anisometropia seen in childhood is transitory. ${ }^{8,27}$ Children at age 12 years with anisometropia may not necessarily have been anisometropic at age 6 years, with the converse also applying. Prospective follow-up data will help describe the change in anisometropia with age and its implication for visual function, including whether anisometropia and aniso-astigmatism precede hyperopia or occur as a result of it, and a review of our study's participants is underway.

The finding of an association of anisometropia with increased interocular differences in axial length, but not corneal curvature, is similar to other studies. ${ }^{14,25}$ However, the Sydney Myopia Study also reports a statistically significant association between the prevalence of anisometropia and increased interocular variation in ACD measures (using the Zeiss IOLMaster) that was not found in our study. The reproducibility of using this instrument to measure ACD has been questioned previously, ${ }^{28}$ as it relies on a subjective judgment by the operator and may explain the difference in findings. Aniso-astigmatism is associated not only with an increased interocular difference in corneal astigmatism, but also with axial length, possibly due to the relationship among aniso-astigmatism, hyperopia, and anisometropia.

It is difficult to explain how similar genetic and environmental influences cause an interocular difference in axial length growth. To date much of the research on anisometropia has concentrated primarily on the mechanism behind amblyopia development. ${ }^{4}$ Although there is a clear association between strabismus and anisometropia, ${ }^{9}$ with ocular dominance ${ }^{29}$ and premature birth influencing anisometropia development, ${ }^{30}$ no definitive biologic or environmental risk factors have been identified currently to our knowledge. ${ }^{9}$ However it has been shown recently that spherical equivalent refractive error decreases to less hyperopia in the amblyopic eye of children who have had treatment for their amblyopia, and this emmetropization process is greatest in children with better ocular alignment. ${ }^{31}$ It has been postulated that neural binocular coordination may be incomplete in anisometropia, ${ }^{26}$ and it recently has been proposed that anisometropia may occur as a result of children adopting a reading position that results in an asymmetric working distance for the two eyes. This, in turn,

TABLE 4. Prevalence Data for Distance Binocular Vision Status (All Subjects)

\begin{tabular}{lcc}
\hline & \multicolumn{2}{c}{ Prevalence \% (95\% CI) } \\
\cline { 2 - 3 } & \multicolumn{1}{c}{ Age 6 to 7 Years } & Age 12 to 13 Years \\
\hline Orthophoria & $92.8(89.5-96.1), n=361$ & $92.2(89.5-94.9), n=606$ \\
Esotropia & $2.1(0.9-3.2), n=8$ & $2.4(1.3-3.5), n=16$ \\
Exotropia & $1.0(0.0-2.3), n=4$ & $0.8(0.2-1.4), n=5$ \\
Heterophoria (eso/exo or hyper) & $4.1(1.8-6.4), n=16$ & $4.6(2.4-6.8), n=30$ \\
\hline
\end{tabular}


would affect markedly the image the peripheral retina receives and the peripheral retina has been implicated in the development of refractive errors. ${ }^{32}$ The association reported by this and other studies between anisometropia and higher spherical refractive errors may suggest that ametropic eyes are less efficient at controlling and coordinating ocular growth.

Anisometropia is an established amblyogenic factor, ${ }^{4}$ and children with greater amounts of anisometropia have a higher prevalence and greater depth of amblyopia. It also is associated with esotropia even in the absence of amblyopia. ${ }^{33}$ In our study, the prevalence of amblyopia was not ascertained, as neither best corrected acuities nor pinhole measures of acuity were made, so the relationship between anisometropia and amblyopia cannot be described. However, the visual acuity and binocular vision data give support to these associations: the prevalence of strabismus is higher in Northern Ireland than that reported in England, where the prevalence in 7-year-old children was $2.3 \%$ (95\% CI, 2.0-2.7). ${ }^{34}$ Although presenting interocular acuity differences were greater in anisometropia and aniso-astigmatism, it is possible that this was due to uncorrected or undercorrected refractive error. Further review of the participants will identify those with amblyopia and help to clarify any associations.

The opinions of professional groups and clinicians vary in terms of the amount of anisometropia that is considered a risk factor for the development of amblyopia. ${ }^{4,35}$ Some investigators promote the use of a different cut-off point for differing types of anisometropia, for example a cut-off value of $>1.00$ DS for hyperopic anisometropia and $>2.00$ DS for myopic anisometropia, as amblyopia occurs more frequently and with lower amounts of anisometropia in hyperopic compared to myopic anisometropes. ${ }^{5}$ The American Association for Pediatric Ophthalmology and Strabismus has introduced a set of standard risk factors that should be detected with preschool screening. These include anisometropia (either spherical or cylindrical) greater than $1.50 \mathrm{D}$ as anisometropic amblyopia is rare, in the absence of strabismus, when anisometropia is less than $1.50 \mathrm{D}$. Although for the majority of patients there is a correlation between the severity of anisometropia and the degree of amblyopia, ${ }^{36}$ Abrahamsson and Sjostrand suggested that the threshold for the development of anisometropic amblyopia may be as high as $3.00 \mathrm{DS}^{37}$ and further studies are required to discern the amount of anisometropia that is amblyogenic in the current population.

\section{Strengths and Limitations}

This paper presents novel data on the profile of anisometropia and aniso-astigmatism in a robustly sampled Northern Ireland population. The participation rate, while lower than expected, compares favorably with the Collaborative Longitudinal Evaluation of Ethnicity and Refractive Error (CLEERE) Study in the United States. ${ }^{38}$ Due to ethical constraints, it is difficult to establish reasons for nonparticipation, and although the ethnicity, sex, and type of schooling of participants are comparable to those of the target population, ${ }^{21}$ participation bias cannot be excluded. Although this is a school-based rather than a population-based sample, education is compulsory for children in Northern Ireland until 16 years of age, and while official figures are not available it is estimated that less than $1 \%$ of children in Northern Ireland are home-schooled (in the public domain at www.hedni.org/faq).

For comparison with other studies, SER is used to describe the association of anisometropia and aniso-astigmatism with refractive error. In this population there is a high prevalence of astigmatism, which results in some individuals with astigma- tism being classified as myopic. However, using the most ametropic meridian in either eye to classify refractive error did not change the associations with anisometropia and refractive status described within this population.

\section{Conclusions}

There is a high prevalence of axial anisometropia and corneal/ axial aniso-astigmatism in school children in Northern Ireland. At age 12 to 13 years, anisometropia and aniso-astigmatism are more common in children with moderate hyperopia, but whether these relations are causal currently is unclear. The developmental mechanisms underlying anisometropia and aniso-astigmatism must be explored further.

\section{References}

1. Rabin J, Bradley A, Freeman RD. On the relation between aniseikonia and axial anisometropia. Am J Optom Physiol Opt. 1983;60:553-558.

2. Weakley DR Jr, Birch E, Kip K. The role of anisometropia in the development of accommodative esotropia. J AAPOS. 2001;5: 153-157.

3. Weakley DR. The association between anisometropia, amblyopia, and binocularity in the absence of strabismus. Trans Am Ophthalmol Soc. 1999;97:987-1021.

4. Donahue SP. The relationship between anisometropia, patient age, and the development of amblyopia. Trans Am Ophthalmol Soc. 2005;103:313-336.

5. Dobson V, Miller JM, Clifford-Donaldson CE, Harvey EM. Associations between anisometropia, amblyopia, and reduced stereoacuity in a school-aged population with a high prevalence of astigmatism. Invest Ophthalmol Vis Sci. 2008;49: 4427-4436.

6. Wood IC, Hodi S, Morgan L. Longitudinal change of refractive error in infants during the first year of life. Eye (Lond). 1995; 9(Pt 5):551-557.

7. Mayer DL, Hansen RM, Moore BD, Kim S, Fulton AB. Cycloplegic refractions in healthy children aged 1 through 48 months. Arch Ophthalmol. 2001;119:1625-1628.

8. Abrahamsson M, Fabian G, Sjostrand J. A longitudinal study of a population based sample of astigmatic children. II. The changeability of anisometropia. Acta Ophthalmol (Copenh). 1990;68:435-440.

9. Borchert M, Tarczy-Hornoch K, Cotter SA, et al. Anisometropia in Hispanic and African American infants and young children the multi-ethnic pediatric eye disease study. Ophthalmology. 2010;117:148-153.e1.

10. de Vries J. Anisometropia in children: analysis of a hospital population. Br J Ophthalmol. 1985;69:504-507.

11. Dobson V, Harvey EM, Miller JM, Clifford-Donaldson CE. Anisometropia prevalence in a highly astigmatic school-aged population. Optom Vis Sci. 2008;85:512-519.

12. Deng L, Gwiazda JE. Anisometropia in children from infancy to 15 years. Invest Ophthalmol Vis Sci. 2012;53:3782-3787.

13. Tong L, Chan YH, Gazzard G, Tan D, Saw SM. Longitudinal study of anisometropia in Singaporean school children. Invest Ophthalmol Vis Sci. 2006;47:3247-3252.

14. Huynh SC, Wang XY, Ip J, et al. Prevalence and associations of anisometropia and aniso-astigmatism in a population based sample of 6 year old children. Br J Ophthalmol. 2006;90:597601.

15. Shih YF, Hsiao $\mathrm{CH}$, Wen SH, Lin LL, Chen CJ, Hung PT. Prevalence of anisometropia in Taiwanese schoolchildren. $J$ Formos Med Assoc. 2005;104:412-417. 
16. Yamashita T, Watanabe $S$, Ohba N. A longitudinal study of cycloplegic refraction in a cohort of 350 Japanese schoolchildren. anisometropia. Ophthalmic Physiol Opt. 1999;19:30-33.

17. French AN, O'Donoghue L, Morgan IG, Saunders KJ, Mitchell P, Rose KA. Comparison of refraction and ocular biometry in European Caucasian children living in Northern Ireland and Sydney, Australia. Invest Ophthalmol Vis Sci. 2012;53:40214031.

18. O'Donoghue L, Rudnicka AR, McClelland JF, Logan NS, Owen CG, Saunders KJ. Refractive and corneal astigmatism in white school children in Northern Ireland. Invest Ophthalmol Vis Sci. 2011;52:4048-4053.

19. O'Donoghue L, McClelland JF, Logan NS, Rudnicka AR, Owen CG, Saunders KJ. Refractive error and visual impairment in school children in Northern Ireland. Br J Ophthalmol. 2010; 94:1155-1159.

20. Logan NS, Shah P, Rudnicka AR, Gilmartin B, Owen CG. Childhood ethnic differences in ametropia and ocular biometry: The Aston eye study. Ophthalmic Physiol Opt. 2011;31:550-558.

21. O'Donoghue L, Saunders KJ, McClelland JF, et al. Sampling and measurement methods for a study of childhood refractive error in a UK population. Br J Ophthalmol. 2010;94:11501154.

22. Huynh SC, Kifley A, Rose KA, Morgan I, Heller GZ, Mitchell P. Astigmatism and its components in 6-year-old children. Invest Ophthalmol Vis Sci. 2006;47:55-64.

23. Qin XJ, Margrain TH, To CH, Bromham N, Guggenheim JA. Anisometropia is independently associated with both spherical and cylindrical ametropia. Invest Ophthalmol Vis Sci. 2005; 46:4024-4031.

24. Huynh SC, Kifley A, Rose KA, Morgan IG, Mitchell P. Astigmatism in 12-year-old Australian children: comparisons with a 6-year-old population. Invest Ophthalmol Vis Sci. 2007; 48:73-82.

25. Tong L, Saw SM, Lin Y, Chia KS, Koh D, Tan D. Incidence and progression of astigmatism in Singaporean children. Invest Ophthalmol Vis Sci. 2004;45:3914-3918.

26. Weale RA. On the age-related prevalence of anisometropia. Ophthalmic Res. 2002;34:389-392.
27. Almeder LM, Peck LB, Howland HC. Prevalence of anisometropia in volunteer laboratory and school screening populations. Invest Ophthalmol Vis Sci. 1990;31:2448-2455.

28. Baikoff G, Jitsuo Jodai H, Bourgeon G. Measurement of the internal diameter and depth of the anterior chamber: IOLMaster versus anterior chamber optical coherence tomographer. J Cataract Refract Surg. 2005;31:1722-1728.

29. Linke SJ, Baviera J, Munzer G, Steinberg J, Richard G, Katz T. Association between ocular dominance and spherical/ astigmatic anisometropia, age, and sex: analysis of 10,264 myopic individuals. Invest Ophthalmol Vis Sci. 2011;52: 9166-9173.

30. Saunders KJ, McCulloch DL, Shepherd AJ, Wilkinson AG. Emmetropisation following preterm birth. Br J Ophthalmol. 2002;86:1035-1040.

31. Kulp MT, Foster NC, Holmes JM, et al. Effect of ocular alignment on emmetropization in children $<10$ years with amblyopia. Am J Ophthalmol. 2012;154:297-302.e1.

32. Charman WN. Myopia, posture and the visual environment. Ophthalmic Physiol Opt. 2011;31:494-501.

33. Weakley DR Jr, Birch E. The role of anisometropia in the development of accommodative esotropia. Trans Am Ophthalmol Soc. 2000;98:71-76, discussion 76-79.

34. Williams C, Northstone K, Howard M, Harvey I, Harrad RA, Sparrow JM. Prevalence and risk factors for common vision problems in children: data from the ALSPAC study. $\mathrm{Br} J$ Ophthalmol. 2008;92:959-964.

35. Saunders KJ. Early refractive development in humans. Surv Ophthalmol. 1995;40:207-216.

36. Caputo R, Frosini R, De Libero C, Campa L, Magro EF, Secci J. Factors influencing severity of and recovery from anisometropic amblyopia. Strabismus. 2007;15:209-214.

37. Abrahamsson M, Sjostrand J. Natural history of infantile anisometropia. Br J Ophthalmol. 1996;80:860-863.

38. Zadnik K, Manny RE, Yu JA, et al. Collaborative Longitudinal Evaluation of Ethnicity and Refractive Error (CLEERE) Study Group. Ocular component data in schoolchildren as a function of age and gender. Optom Vis Sci. 2003;80:226-36. 